\title{
Stem cells in the context of evolution and development
}

\author{
Volker Hartenstein
}

Received: 2 November 2012 / Accepted: 12 November 2012 / Published online: 6 December 2012

(C) Springer-Verlag Berlin Heidelberg 2012

The field of stem cell biology originated and grew around the discovery that it was possible to isolate undifferentiated cells ("stem cells") from various adult organs, as well as from early mammalian embryos. These could then be grown in culture, made to differentiate into various cell types, and put back into host organisms. It gradually became clear that most, if not all, adult tissues possess stem cells, and that neoplastic growth typically takes off from stem cells. Since the implications for the cure of cancer and degenerative diseases are great, research on the biology of stem cells has expanded rapidly over the past two decades. In particular, stem cell research has moved into using genetic model systems, including the invertebrates Drosophila and Caenorhabditis elegans, and into investigations of stem cell development (in vertebrates and Drosophila alike). Both approaches have delivered significant new insights. In small organisms such as Drosophila, stem cell pools and their niche environment often consist of only a few cells, and questions concerning the pattern of cell division, niche-stem cell contacts, or differentiation can be addressed at the single cell level (as for example, in the gonadal stem cells and the intestine; Spradling et al. 2011; Eliazer and Buszczak 2011; Papagiannouli and Lohmann 2012; Jiang and Edgar 2012; Takashima and Hartenstein 2012). Studying the development of stem cells offers the opportunity to follow step by step where in the embryo these cells come from, what structures in their environment they interact with, and what signaling pathways are active sequentially as a result of these interactions.

\section{Hartenstein $(\triangle)$}

Department of Biology, University of California Los Angeles,

621 Charles E. Young Dr,

Los Angeles, CA 90095-1606, USA

e-mail: volkerh@mcdb.ucla.edu
Stem cells of the adult organism are typically slowly cycling, undifferentiated, and often multipotent cells located in special microenvironments, called "niches" (MartinezAgosto et al. 2007; Haegebarth and Clevers 2009; Boral and Nie 2012). Embryonic stem cells can be molecularly defined by a small number of transcription factors and signaling pathways (Boyer et al. 2006; Loh et al. 2008; Chambers and Tomlinson 2009) which are crucial for self renewal, and which are even able to induce embryonic stem (ES) cell characteristics when expressed in mature cells. The combination of "ES stemness factors" found in ES cells is not typically expressed in adult stem cells of vertebrates, or other model organisms; instead, adult somatic stem cells typically express markers specific for an individual organ or tissue (e.g., membrane glycoprotein CD34 in hematopoietic stem cells; Lgr5 in intestinal stem cells; Leushacke and Barker 2012). Through their mitotic activity, stem cells generate two types of offspring. First, they renew themselves and thereby maintain a pool of proliferating stem cells. Secondly, they produce offspring that then become postmitotic and differentiate or (more typically) that first undergo a phase of rapid cell division before differentiating. Since these cells have a limited proliferative potential and eventually turn into differentiated progeny, they are referred to as transient amplifying cells.

The great interest in stem cells, whether analyzed in a culture system or in model organisms, has also spawned lines of research that aim at the evolutionary origin and subsequent modifications of this fundamental cell type. Many areas of early research received an infusion of new concepts and new technologies. Hydrozoa and Planaria, to name but two examples, have long been known for their amazing capacity of regeneration, and the existence of multipotent stem cells (i.e., interstitial cells and neoblasts, respectively) play an important role in regeneration and normal development (Gierer 1977; Chandebois 1976). More 
recently, new technologies have permitted the investigation of refined questions about these stem cells, including questions concerning the signaling pathways controlling their behavior and the batteries of genes that are turned on or off depending on the developmental state (Bosch et al. 2010; Tanaka and Reddien 2011; Baguñà 2012). The quickly expanding field of comparative stem cell biology notably also includes flowering plants, organisms that clearly outdo animals when it comes to vegetative growth (Aichinger et al. 2012). Throughout the ensuing studies, a question that naturally looms in the background is how related, in terms of genetic constitution and developmental potential, are these "primordial stem cell systems" to the stem cells that we observe in highly derived bilaterians? Can one consider multipotent stem cells as fundamentally homologous? Or did they evolve separately from a primitive state that lacked "professional stem cells", where cell renewal occurred by division of differentiated cells, or by dedifferentiation (followed by division) of these cells?

A satisfactory answer to these and many other questions cannot yet be provided, because our knowledge of stem cells outside the few model organisms is far too sketchy. In an effort to bring together information on stem cells by studying multiple different taxa, we collected a set of review articles in this special issue of DGE. In these articles, attention is paid to the questions of developmental origin, lineage potential, and possible homologies of stem cells. The first two articles (Ereskovsky et al. 2013; Funayama 2013) address these issues in sponges. They emphasize the overall conservation in terms of cell movements and morphogenesis that exists between sponges and higher animals and summarize the growing body of evidence for a dual system of stem cells, choanocytes, and archaeocytes as the mechanism underlying growth and regeneration in this animal taxon. The next pair of papers (Galliot 2013; Gold and Jacobs 2013) surveys stem cells of Cnidaria. Hydra, as one member of this taxon, has yielded important insights in the developmental questions of regeneration, organizers, morphogen gradients, and, more recently, specific signaling pathways underlying these phenomena. The article by Galliot summarizes recent research on the interplay between apoptosis, interstitial stem cells, and epithelial stem cells that takes place during injury-induced head regeneration. Gold and Jacobs take a comparative look into the different types of stem cells that have been identified (or affirmed to exist) in Cnidaria outside the hydrozoans. With the paper by Rink (2013) we move into the bilaterian animals, more specifically, the planarians, members of the diverse phylum Platyhelminthes; the review provides an overview of stem cell characteristics that gradually become established for the neoblasts. The last four articles focus on different classes of somatic stem cells, including those of the intestine, blood, heart, and nervous system. Takashima et al. (2013) survey in a comparative manner the developmental mechanisms of producing and maintaining the cell types found in animal guts. Grigorian and Hartenstein (2013) take a look at the process of hematopoiesis in a more restricted set of taxa, the arthropods. The contribution by Pandur et al. (2013) reviews recent data pertaining to the embryonic fields that give rise to tissues, including stem cells, of the vertebrate heart. Finally, Grandel and Brand (2013) focus on the central nervous system of vertebrates, where the fairly restricted distribution and function of neural stem cells found in mammals contrast with the widespread occurrence of these cells in anamniotes; despite this discrepancy, detailed comparisons looking at stem cell development and proliferative mechanisms reveal a number of common themes throughout vertebrates.

We hope the articles of this special issue will be of help as a source of information for researchers in stem cell biology, as well as interested readers who will try to draw together concepts and ideas that are spawned by discoveries in the fields of evolution, development, and stem cells.

\section{References}

Aichinger E, Kornet N, Friedrich T, Laux T (2012) Plant stem cell niches. Annu Rev Plant Biol 63:615-636

Baguñà $J$ (2012) The planarian neoblast: the rambling history of its origin and some current black boxes. Int J Dev Biol 56(1-3):1937

Boral D, Nie D (2012) Cancer stem cells and niche microenvironments. Front Biosci (Elite Ed) 4:2502-2514

Bosch TC, Anton-Erxleben F, Hemmrich G, Khalturin K (2010) The Hydra polyp: nothing but an active stem cell community. Dev Growth Differ 52(1):15-25

Boyer LA, Mathur D, Jaenisch R (2006) Molecular control of pluripotency. Curr Opin Gen Dev 16(5):455-462

Chambers I, Tomlinson SR (2009) The transcriptional foundation of pluripotency. Development 136(14):2311-2322

Chandebois R (1976) Histogenesis and morphogenesis in planarian regeneration. Monogr Dev Biol 11:1-182

Eliazer S, Buszczak M (2011) Finding a niche: studies from the Drosophila ovary. Stem Cell Res Ther 2(6):45. doi:10.1186/ scrt86

Ereskovsky AV, Renard E, Borchiellini C (2013) Cellular and molecular processes leading to embryo formation in sponges: evidences for high conservation of processes throughout animal evolution. Dev Genes Evol. doi:10.007/s00427-0120399-3

Funayama N (2013) The stem cell system in demosponges: suggested involvement of two types of cells: archeocytes (active stem cells) and choanocytes (food-entrapping flagellated cells). Dev Genes Evol. doi:10.007/s00427-012-0417-5

Galliot B (2013) Injury-induced asymmetric cell death as a driving force for head regeneration in Hydra. Dev Genes Evol. doi:10.007/s00427-012-0411-y

Gierer A (1977) Biological features and physical concepts of pattern formation exemplified by hydra. Curr Top Dev Biol 11:17-59 
Gold D, Jacobs D (2013) Stem cell dynamics in Cnidaria: are there unifying principles? Dev Genes Evol. doi:10.1007/s00427-012-0429-1

Grandel H, Brand M (2013) Comparative aspects of adult neural stem cell activity in vertebrates. Dev Genes Evol. doi:10.007/s00427012-0425-5

Grigorian M, Hartenstein V (2013) Hematopoiesis and hematopoietic organs in arthropods. Dev Genes Evol. doi:10.007/s00427-012-0428-2

Haegebarth A, Clevers H (2009) Wnt signaling, lgr5, and stem cells in the intestine and skin. Am J Pathol 174(3):715-721

Jiang H, Edgar BA (2012) Intestinal stem cell function in Drosophila and mice. Curr Opin Genet Dev 22(4):354-360

Leushacke M, Barker N (2012) Lgr5 and Lgr6 as markers to study adult stem cell roles in self-renewal and cancer. Oncogene 31 (25):3009-3022. doi:10.1038/onc.2011.479

Loh YH, Ng JH, Ng HH (2008) Molecular framework underlying pluripotency. Cell Cycle 7(7):885-891

Martinez-Agosto JA, Mikkola HK, Hartenstein V, Banerjee U (2007) The hematopoietic stem cell and its niche: a comparative view. Genes Dev 21(23):3044-3060
Pandur P, Sirbu IO, Kühl SJ, Philipp M, Kühl M (2013) Islet1expressing cardiac progenitor cells: a comparison across species. Dev Genes Evol. doi:10.007/s00427-012-0400-1

Papagiannouli F, Lohmann I (2012) Shaping the niche: lessons from the Drosophila testis and other model systems. Biotechnol J 7 (6):723-736. doi:10.1002/biot.201100352

Rink J (2013) Stem cell systems and regeneration in Planaria. Dev Genes Evol. doi:10.007/s00427-012-0426-4

Spradling A, Fuller MT, Braun RE, Yoshida S (2011) Germline stem cells. Cold Spring Harb Perspect Biol 3(11):a002642. doi:10.1101/cshperspect.a002642

Takashima S, Hartenstein V (2012) Genetic control of intestinal stem cell specification and development: a comparative view. Stem Cell Rev 8(2):597-608

Takashima S, Gold D, Hartenstein V (2013) Stem cells and lineages of the intestine: a developmental and evolutionary perspective. Dev Genes Evol. doi:10.007/s00427-012-0422-8

Tanaka EM, Reddien PW (2011) The cellular basis for animal regeneration. Dev Cell 21(1):172-185 